\title{
Efficacy, acceptability, and safety of antidepressants for low back pain: a systematic review and meta-analysis
}

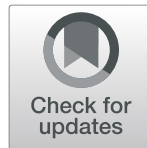

Michael C. Ferraro ${ }^{1,2}$ (D), Matthew K. Bagg ${ }^{1,3,4^{*}}$, Michael A. Wewege ${ }^{1,2}$ (D) Aidan G. Cashin ${ }^{1,3}$ (D), Hayley B. Leake ${ }^{1,5}$, Rodrigo R. N. Rizzo ${ }^{1,2} \mathbb{D}_{\text {, Matthew D. Jones }}^{1,2}$ (D), Sylvia M. Gustin ${ }^{1,6}$, Richard Day ${ }^{7,8}$ (D), Colleen K. Loo ${ }^{9,10}$ (D) and James H. McAuley ${ }^{1,2}$ (D)

\begin{abstract}
Background: Antidepressant medicines are used to manage symptoms of low back pain. The efficacy, acceptability, and safety of antidepressant medicines for low back pain (LBP) are not clear. We aimed to evaluate the efficacy, acceptability, and safety of antidepressant medicines for LBP.

Methods: We searched CENTRAL, MEDLINE, Embase, CINAHL, ClinicalTrials.gov, the EU Clinical Trials Register, and the WHO International Clinical Trial Registry Platform from inception to May 2020. We included published and trial registry reports of RCTs that allocated adult participants with LBP to receive an antidepressant medicine or a placebo medicine. Pairs of authors independently extracted data in duplicate. We extracted participant characteristics, study sample size, outcome values, and measures of variance for each outcome. We data using random-effects meta-analysis models and calculated estimates of effects and heterogeneity for each outcome. We formed judgments of confidence in the evidence in accordance with GRADE. We report our findings in accordance with the PRISMA statement. We prespecified all outcomes in a prospectively registered protocol. The primary outcomes were pain intensity and acceptability. We measured pain intensity at end-of-treatment on a 0-100 point scale and considered 10 points the minimal clinically important difference. We defined acceptability as the odds of stopping treatment for any reason.

Results: We included 23 RCTs in this review. Data were available for pain in 17 trials and acceptability in 14 trials. Treatment with antidepressants decreased pain intensity by 4.33 points $(95 \% \mathrm{Cl}-6.15$ to -2.50$)$ on a $0-100$ scale, compared to placebo. Treatment with antidepressants increased the odds of stopping treatment for any reason (OR 1.27 [95\% Cl 1.03 to 1.56$])$, compared to placebo.

(Continued on next page)
\end{abstract}

\footnotetext{
* Correspondence: m.bagg@neura.edu.au

'Centre for Pain IMPACT, Neuroscience Research Australia, Sydney, NSW, Australia

${ }^{3}$ Prince of Wales Clinical School, University of New South Wales, Sydney, Australia

Full list of author information is available at the end of the article
}

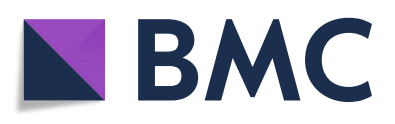

(C) The Author(s). 2021 Open Access This article is licensed under a Creative Commons Attribution 4.0 International License, which permits use, sharing, adaptation, distribution and reproduction in any medium or format, as long as you give appropriate credit to the original author(s) and the source, provide a link to the Creative Commons licence, and indicate if changes were made. The images or other third party material in this article are included in the article's Creative Commons licence, unless indicated otherwise in a credit line to the material. If material is not included in the article's Creative Commons licence and your intended use is not permitted by statutory regulation or exceeds the permitted use, you will need to obtain permission directly from the copyright holder. To view a copy of this licence, visit http://creativecommons.org/licenses/by/4.0/ The Creative Commons Public Domain Dedication waiver (http://creativecommons.org/publicdomain/zero/1.0/) applies to the data made available in this article, unless otherwise stated in a credit line to the data. 
(Continued from previous page)

Conclusions: Treatment of LBP with antidepressants is associated with small reductions in pain intensity and increased odds of stopping treatment for any reason, compared to placebo. The effect on pain is not clinically important. The effect on acceptability warrants consideration. These findings provide Level I evidence to guide clinicians in their use of antidepressants to treat LBP.

Trial registration: We prospectively registered the protocol for this systematic review on PROSPERO (CRD4202014 9275).

Keywords: Low back pain, Antidepressants, Analgesics, Drug therapy, Review, Meta-analysis

\section{Background}

Low back pain (LBP) is the leading cause of disability worldwide [1]. The most common interventions for LBP are medicines that aim to reduce symptoms [2-7]. Clinical guidelines for LBP recommend that medicines should be prescribed for those who fail to respond to non-pharmacological interventions [8-11] and restricted to short-term use due to the potential for adverse effects and abuse [11]. Common medicines prescribed for LBP include non-steroidalantiinflammatories (NSAIDs), opioids, muscle relaxants, and antidepressants [3, 12-14].

Antidepressants are a broad group of medicines classified according to their presumed action [15]. The mechanism of their analgesic effects is not well understood $[16,17]$. Antidepressants are prescribed for LBP to provide pain relief, improve sleep, or reduce co-morbid depressive symptoms [18]. There is evidence that prescription rates of antidepressants to manage LBP are increasing $[14,19]$.

Evidence to support the efficacy and safety of antidepressants for LBP is unclear. Findings from systematic reviews are inconsistent [20-23]. The most recent review found inconclusive evidence for the effect of antidepressant medicines on pain intensity, disability or depression [23], and inadequate evidence to evaluate the acceptability and safety of antidepressants for LBP. The most recently published clinical guidelines for LBP provide conflicting advice on the use of antidepressants for LBP. The American College of Physicians guideline endorses duloxetine for chronic LBP [11] whereas the National Institute for Health and Care Excellence (UK) guideline advises against the use of any antidepressant for LBP [9].

The aim of this systematic review was to evaluate the efficacy, acceptability, and safety of antidepressant medicines compared to placebo for LBP, using data from published and trial registry reports.

\section{Methods}

We prospectively registered the protocol [24] for this systematic review on PROSPERO (CRD42020149275) and report our findings according to the Preferred
Reporting Items for Systematic Reviews and MetaAnalyses (PRISMA) guideline [25] (Checklist S1 in Additional file 1).

\section{Primary outcomes}

The primary outcomes were pain intensity and acceptability. Pain intensity was measured at the follow-up assessment closest to the end of treatment. Acceptability, defined as overall acceptability of the medicine, was measured using all-cause discontinuation during treatment $[15,26]$.

\section{Secondary outcomes}

The secondary outcomes included low back-specific function, symptoms of depression, safety, harm, and tolerability. Low back-specific function and symptoms of depression were measured at the follow-up assessment closest to the end of treatment. Safety and harm, defined as the incidence of adverse effects and serious adverse effects [27], were measured by reports of adverse effects and serious adverse effects during treatment. Tolerability was defined as the tolerability of adverse effects sustained during treatment, measured by reports of discontinued treatment due to adverse effects.

\section{Data sources}

We used comprehensive search strategies to search electronic databases and clinical trial registries for records of randomized clinical trials of antidepressant medicines in LBP (Appendix S1 in Additional file 2) [28, 29]. We piloted the strategies using records of trials included in a previous systematic review [23]. We searched the Cochrane Back and Neck Group's Trials Register and the Cochrane Central Register of Controlled Trials (CENTRAL) (Cochrane Library), MEDLINE, Embase (Ovid), and CINAHL (EBSCO) databases from inception to May 15, 2020. We searched ClinicalTrials.gov (ClinicalTrials.gov), the EU Clinical Trials Register (www.clinicaltrialsregister.eu), and the WHO International Clinical Trial Registry Platform (apps.who.int/ trialsearch/Default.aspx) from inception to May 15, 2020. We included records written in English, Italian, Spanish, Portuguese, German, and French. 
We included published and trial registry reports of randomized controlled trials (RCTs) that allocated adult participants with LBP to receive (i) a systemically administered dose of an antidepressant medicine or (ii) a sham (placebo) medicine, (iii) continuation of usual care, (iv) a waiting list, or (v) no-treatment. LBP was defined as pain of any duration between the 12th rib and buttock crease, with or without associated leg pain [30]. Trials that only included participants with symptoms of nerve root compromise (sciatica) [31] or LBP due to specific medical conditions (e.g., spinal fracture, inflammatory disease, aortic dissection, malignancy, or infection) were excluded. We included trials of mixed samples (e.g., nonspecific LBP and LBP with sciatica, or non-specific LBP and large joint osteoarthritis) if separate data for the non-specific LBP sample were available. We included trials that tested the efficacy of selective serotonin reuptake inhibitors (SSRIs), serotonin and norepinephrine reuptake inhibitors (SNRIs), tricyclic antidepressants (TCAs), tetracyclic antidepressants (TeCA), heterocyclic antidepressants (HCAs), monoamine oxidase inhibitors (MAOIs), or atypical antidepressants, provided they were listed on the WHO ATC [32] and licensed in at least one of the following jurisdictions: USA (FDA) [33], Australia (TGA) [34], UK (MHRA) [35], or Europe (EMA) [36].

We screened records for inclusion in two stages. Pairs of authors from a team of six (MCF, MAW, AGC, MDJ, HBL, RRNR) independently screened record titles and abstracts in duplicate. The full texts of potentially eligible records were retrieved and independently screened again (MCF, MAW) to confirm inclusion. Disagreements were resolved through discussion or recourse to a third author (MKB or JHM).

We linked records to identify unique studies using a hierarchy. Records that were published and reported the results of a trial were classified as primary records, followed by other published records of a trial (e.g., secondary analyses), conference abstracts, and lastly, trial registry records. We classified the trial registry record as the primary record if there was no evidence of publication.

\section{Data extraction and risk of bias assessment}

Pairs of authors (MCF, MAW, AGC, HBL, RRNR, and MDJ) independently extracted data using standardized, piloted, data extraction forms and assessed study-level risk of bias using the Cochrane "Risk of bias" tool (version 5.1.0) [37] and published recommendations [38, 39]. Outcomes were rated as low overall risk when three or fewer domains are rated "unclear" risk, and no domains were rated "high"; moderate risk if a single domain was rated as "high" risk, but four or more were rated as "unclear" and high overall risk in all other instances. We resolved conflicts by consensus or, where necessary, through arbitration with a third author (MKB, JHM). We extracted, for each trial, the following: participant age, sex, duration of symptoms, and sample size; outcome value and measure of variance for pain intensity, function, and symptoms of depression; number of adverse and serious adverse effects; and the number of participants that discontinued treatment for any reason or due to adverse effects. We used an established hierarchy to preference data from continuous measures of pain, function, and symptoms of depression and converted all outcome data to a $0-100$-point scale [24]. We used recommended methods $[40,41]$ to calculate standard deviations when these were not available.

\section{Effect measures and interpretation}

We used the difference in means and accompanying 95\% confidence intervals for analyses of effects of antidepressant medicines on continuous outcomes (pain, function, symptoms of depression). We followed recommended guidance for trials with multiple arms by dividing the control group sample size by the number of arms in the study (Cochrane Handbook, Version 6) [42]. For cross-over trials where we were unable to obtain the first phase outcome data from the study authors, we included the overall effect (reflecting both phases) adjusted to correct for the correlation between the two phases [41]. The minimal clinically important difference in means is established as 10 points on a common 0-100point scale for both pain and function [42]. We used the odds ratio and accompanying 95\% confidence intervals for analyses of effects of antidepressant medicines on binary outcomes (acceptability, safety, harm, tolerability).

\section{Data synthesis \\ Main analysis}

We synthesized the data for each outcome using frequentist random-effectsmeta-analysis models. We fit the models using Restricted Maximum Likelihood (REML) in the $\mathrm{R}$ (version 3.6.2) package metafor (version 2.4-0) $[43,44]$. We calculated the Q statistic to estimate heterogeneity, the estimate of between-study variance $\left(\tau^{2}\right)$, and the proportion of this variance not due to sampling error $\left(I^{2}\right)$. We calculated the $95 \%$ prediction interval for the pooled effect and displayed this on the forest plot alongside the pooled effect estimate and 95\% confidence interval.

\section{Investigation of heterogeneity}

We specified symptom duration, medicine type, and dose as covariates for investigation of important heterogeneity in the main analyses. Symptom duration had three levels: 0-6 weeks, 6-12 weeks, and > 12 weeks. Medicine type had seven levels: atypical, HCA, MAOI, 
SSRI, SNRI, TCA, TeCA. We included an additional level of medicine dose, compared to the protocol: standard dose range (SDR), less than SDR, and above SDR according to the Prescriber's Digital Reference [45]. We conducted subgroup analyses, using the covariate levels as strata.

\section{Sensitivity analyses}

We tested the effect of the definition of non-specific LBP and of imputing missing measures of variance by repeating the main analyses with and without the relevant studies.

\section{Influence of a hypothetical RCT}

We constructed extended funnel plots using Stata (version 14.2) [46] to simulate the influence of hypothetical parameters of a future RCT on the pooled effect estimate for pain intensity $[47,48]$. The extended funnel plot augments a funnel plot with overlays to provide an illustration of the impact of a new trial on a given metaanalysis [48]. We used 10 points on a $0-100$ pain intensity scale as the threshold for the smallest worthwhile effect. We did not perform this analysis for acceptability as there is no known smallest worthwhile effect for this outcome.

\section{Confidence in cumulative evidence}

Two authors (MCF, MAW) used the Grading of Recommendations Assessment Development and Evaluation (GRADE) [49] framework to develop judgements of high, moderate, low, or very low confidence in the evidence for each outcome. We assessed the domains of study limitations, inconsistency, imprecision, and publication bias, using planned criteria [24]. Publication bias was evaluated using visual assessment of funnel plot symmetry, and Egger's tests where 10 or more studies were available for an outcome [50].

\section{Results}

\section{Search results}

The search identified 2598 records. We removed 371 duplicates and screened the titles and abstracts of 2227 records for inclusion. We excluded 2104 records and retrieved the full-texts of 123 potentially eligible records

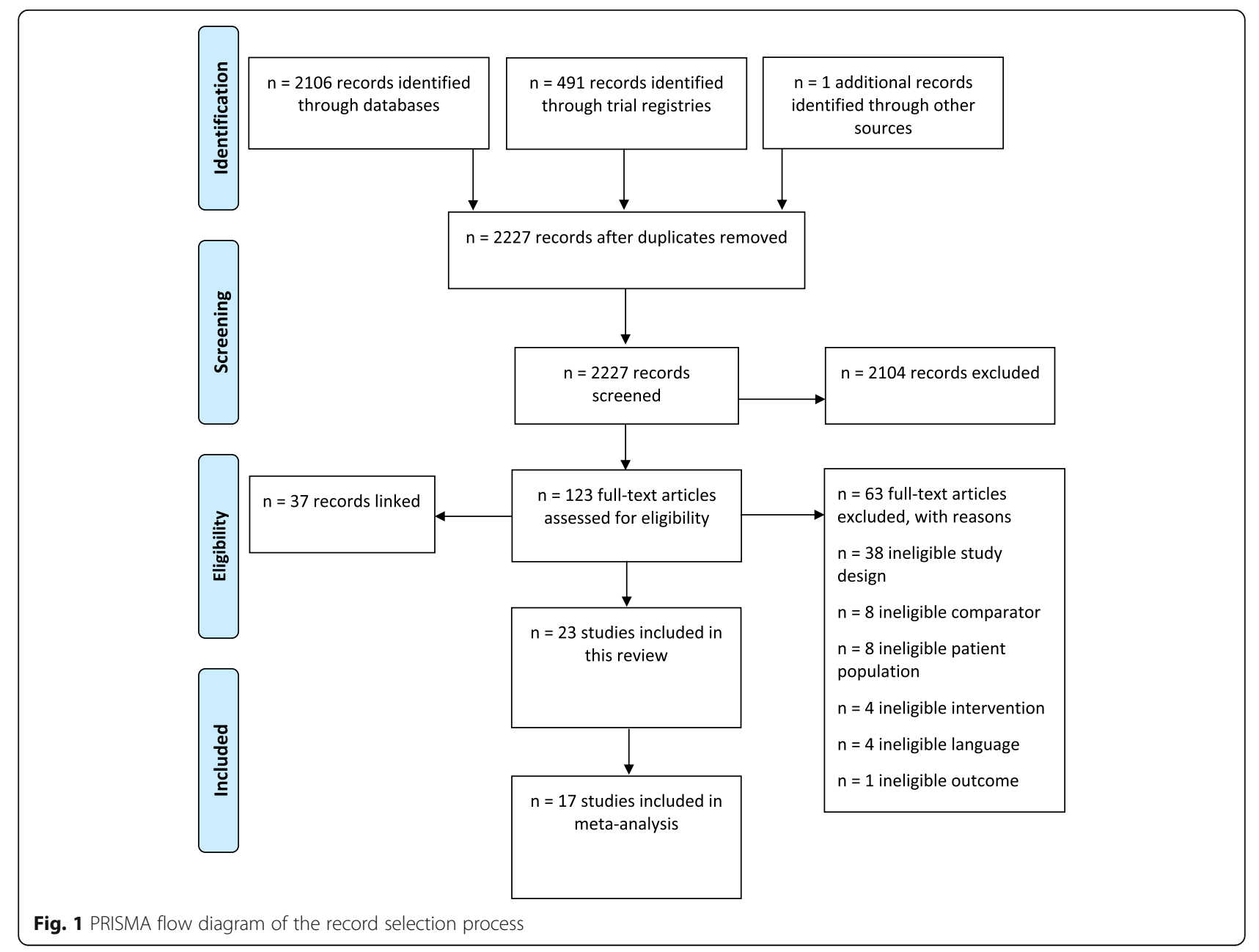


Table 1 Characteristics of included studies

\begin{tabular}{|c|c|c|c|c|c|c|c|}
\hline $\begin{array}{l}\text { Label, } \\
\text { citation }\end{array}$ & $\begin{array}{l}\text { Study level data unless } \\
\text { reported otherwise; } \\
\text { mean (SD) }\end{array}$ & Setting & $\begin{array}{l}\text { Number } \\
\text { of trial } \\
\text { arms }\end{array}$ & $\begin{array}{l}\text { Intervention, number } \\
\text { assigned } \\
\text { (mg/day unless } \\
\text { indicated) }\end{array}$ & $\begin{array}{l}\text { Comparator, number } \\
\text { assigned } \\
\text { (mg/day unless } \\
\text { indicated) }\end{array}$ & $\begin{array}{l}\text { Duration } \\
\text { of } \\
\text { treatment }\end{array}$ & $\begin{array}{l}\text { Outcome } \\
\text { measures } \\
\text { applicable } \\
\text { to this } \\
\text { review }\end{array}$ \\
\hline $\begin{array}{l}\text { Alcoff et al. } \\
\text { [51] }\end{array}$ & $\begin{array}{l}50 \text { participants with } \\
\text { subacute and chronic LBP; } \\
\text { mean age imipramine } \\
\text { group } 29.2 \text { years, placebo } \\
\text { group } 33.8 \text { years }{ }^{\text {b }} n=24 \\
(48 \%) \text { female }\end{array}$ & USA; 2 sites & 2 & $\begin{array}{l}\text { Oral imipramine } 75 \text { for } 3 \\
\text { days, } 150 \text { thereafter, } \\
n=28\end{array}$ & Placebo, $n=22$ & 8 weeks & $\mathrm{SBPQ}, \mathrm{BDI}$ \\
\hline $\begin{array}{l}\text { Atkinson et al. } \\
\text { [52] }\end{array}$ & $\begin{array}{l}121 \text { participants with } \\
\text { chronic LBP; mean age } \\
46.4 \text { (10.2) years; } n=47 \\
(38.8 \%) \text { female }\end{array}$ & USA & 7 & $\begin{array}{l}\text { Oral desipramine target } \\
\text { concentrations of } 50 \mathrm{ng} / \\
\mathrm{mL} n=17 \text {, or } 110 \mathrm{ng} / \mathrm{mL} \\
n=17 \text {, or } 150 \mathrm{ng} / \mathrm{mL} \\
n=18 \text {, or fluoxetine target } \\
\text { concentrations of } 50 \mathrm{ng} / \\
\mathrm{mL} n=14, \text { or } 100 \mathrm{ng} / \mathrm{mL} \\
n=14 \text {, or } 150 \mathrm{ng} / \mathrm{mL} \\
n=15\end{array}$ & $\begin{array}{l}\text { Active placebo } \\
\text { (benztropine mesylate) } \\
n=26\end{array}$ & 12 weeks & $\begin{array}{l}\text { DDS, BDI, } \\
\text { RMDQ }\end{array}$ \\
\hline $\begin{array}{l}\text { Atkinson et al. } \\
\text { [62] }\end{array}$ & $\begin{array}{l}103 \text { participants with } \\
\text { chronic LBP; mean age } \\
49.2 \text { ( } 9.4 \text { ) years; } n=38 \\
\text { (37\%) female }\end{array}$ & USA & 3 & $\begin{array}{l}\text { Oral maprotiline } 150 \\
n=33 \text {, or paroxetine } 30 \\
n=34\end{array}$ & $\begin{array}{l}\text { Active placebo } \\
\text { (diphenhydramine) } \\
37.5, n=36\end{array}$ & 8 weeks & DDS, BDI \\
\hline $\begin{array}{l}\text { Atkinson et al. } \\
\text { [63] }\end{array}$ & $\begin{array}{l}78 \text { participants with } \\
\text { chronic LBP; mean age } \\
\text { nortriptyline group } 45.79 \\
\text { (10.59) years, placebo } \\
\text { group } 47.13 \text { (10.65) years; } \\
n=0(0 \%) \text { female }\end{array}$ & USA & 2 & $\begin{array}{l}\text { Oral nortriptyline } 25 \text { for } 3 \\
\text { days, } 50 \text { for } 4 \text { days, } 75 \text { for } \\
3 \text { days, } 100 \text { for } 4 \text { days to } \\
\text { reach target concentration } \\
\text { of } 50-150 \mathrm{ng} / \mathrm{ml}, n=38\end{array}$ & Placebo, $n=40$ & 8 weeks & DDS, BDI \\
\hline $\begin{array}{l}\text { Dickens et al. } \\
\text { [64] }\end{array}$ & 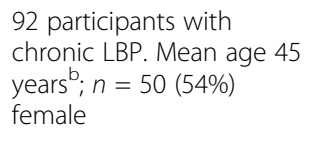 & UK & 2 & $\begin{array}{l}\text { Oral paroxetine } 20 \\
n=44\end{array}$ & Placebo, $n=48$ & 8 weeks & $\begin{array}{l}100 \mathrm{~mm} \\
\text { VAS, MADR } \\
\mathrm{S}\end{array}$ \\
\hline $\begin{array}{l}\text { Goodkin et al. } \\
\text { [65] }\end{array}$ & $\begin{array}{l}42 \text { participants with } \\
\text { chronic LBP; mean age } \\
53.6 \text { (12.9) years; } n=16 \\
\text { (38\%) female }\end{array}$ & USA & 2 & $\begin{array}{l}\text { Oral trazodone } 50, \\
\text { increasing to } 600, n=22\end{array}$ & Placebo, $n=22$ & 6 weeks & $\begin{array}{l}100 \mathrm{~mm} \\
\text { VAS, BDI }\end{array}$ \\
\hline $\begin{array}{l}\text { Gould et al. } \\
\text { [66] }\end{array}$ & $\begin{array}{l}142 \text { participants with } \\
\text { chronic LBP; mean age } \\
55.8 \text { (11.7) years; } n=15 \\
(11 \%) \text { female }\end{array}$ & USA & 4 & $\begin{array}{l}\text { Oral desipramine } \\
\text { hydrochloride to reach } \\
\text { target concentration of } \\
5-60 \mathrm{ng} / \mathrm{ml}, n=37 \text {, or } \\
\text { desipramine hydrochloride } \\
\text { to reach target } \\
\text { concentration of } 5-60 \mathrm{ng} / \\
\mathrm{ml} \text { and cognitive } \\
\text { behavioral therapy, } n=37\end{array}$ & $\begin{array}{l}\text { Active placebo } \\
\text { (benztropine mesylate) } \\
0.125 \text { and cognitive } \\
\text { behavioral therapy, } \\
n=33 \text {, or active } \\
\text { placebo (benztropine } \\
\text { mesylate) } 0.125, n=32\end{array}$ & 12 weeks & $\begin{array}{l}\text { DDS, } \\
\text { RMDQ }\end{array}$ \\
\hline $\begin{array}{l}\text { Jenkins et al. } \\
\text { [67] }\end{array}$ & $\begin{array}{l}59 \text { participants with acute } \\
\text { and chronic LBP; mean } \\
\text { age imipramine group } 26 \\
\text { years, placebo group } 26.7 \\
\text { years }^{b} ; n=3(5 \%) \text { female }\end{array}$ & UK & 2 & Oral imipramine $75, n=30$ & Placebo, $n=29$ & 4 weeks & $\begin{array}{l}10 \mathrm{~cm} \text { VAS, } \\
\mathrm{BDI}\end{array}$ \\
\hline $\begin{array}{l}\text { Johnson et al. } \\
\text { [68] } \\
\text { (crossover) }\end{array}$ & $\begin{array}{l}14 \text { participants with } \\
\text { chronic LBP; mean age } \\
36.93 \text { (13.05) years; } n=0 \\
\text { (0\%) female }\end{array}$ & USA & 2 & $\begin{array}{l}\text { Oral duloxetine, } 30 \text { for } 1 \\
\text { week, titration to } 60 \text { for } 2 \\
\text { weeks, then maintenance } \\
\text { for } 4 \text { weeks, } 30 \text { for final } \\
\text { week, } n=7^{\text {a }}\end{array}$ & Placebo, $n=7^{a}$ & $\begin{array}{l}8 \text { weeks/ } \\
\text { phase } \\
\text { with 1- } \\
\text { week } \\
\text { washout }\end{array}$ & BPI \\
\hline $\begin{array}{l}\text { Katz et al. [69] } \\
\text { (crossover) }\end{array}$ & $\begin{array}{l}54 \text { participants with } \\
\text { chronic LBP; mean age } \\
50.6 \text { (10.7) years; } n=21 \\
(48 \%) \text { female }^{c}\end{array}$ & USA & 2 & $\begin{array}{l}\text { Oral bupropion } 150 \text { for } 3 \\
\text { days, } 300 \text { until end week } \\
5,150 \text { until week } 7 \text {, } \\
n=21^{\text {a }}\end{array}$ & Placebo, $n=23^{\mathrm{a}}$ & $\begin{array}{l}7 \text { weeks/ } \\
\text { phase } \\
\text { with 2- } \\
\text { week } \\
\text { washout }\end{array}$ & $\begin{array}{l}\text { 11-point } \\
\text { NRS, BDI, } \\
\text { RMDQ }\end{array}$ \\
\hline
\end{tabular}


Table 1 Characteristics of included studies (Continued)

\begin{tabular}{|c|c|c|c|c|c|c|c|}
\hline $\begin{array}{l}\text { Study } \\
\text { Label, } \\
\text { citation }\end{array}$ & $\begin{array}{l}\text { Patient sample } \\
\text { Study level data unless } \\
\text { reported otherwise; } \\
\text { mean (SD) }\end{array}$ & Setting & $\begin{array}{l}\text { Number } \\
\text { of trial } \\
\text { arms }\end{array}$ & $\begin{array}{l}\text { Intervention, number } \\
\text { assigned } \\
\text { (mg/day unless } \\
\text { indicated) }\end{array}$ & $\begin{array}{l}\text { Comparator, number } \\
\text { assigned } \\
\text { (mg/day unless } \\
\text { indicated) }\end{array}$ & $\begin{array}{l}\text { Duration } \\
\text { of } \\
\text { treatment }\end{array}$ & $\begin{array}{l}\text { Outcome } \\
\text { measures } \\
\text { applicable } \\
\text { to this } \\
\text { review }\end{array}$ \\
\hline $\begin{array}{l}\text { Konno et al. } \\
\text { [53] }\end{array}$ & $\begin{array}{l}458 \text { participants with } \\
\text { chronic LBP; mean age } \\
58.9 \text { (13.4) years; } n=237 \\
(52 \%) \text { female }\end{array}$ & $\begin{array}{l}\text { Japan; } 58 \\
\text { sites }\end{array}$ & 2 & $\begin{array}{l}\text { Oral duloxetine } 20 \text { first } \\
\text { week, } 40 \text { second week, } 60 \\
\text { weeks } 3-14, n=232\end{array}$ & Placebo, $n=226$ & 14 weeks & $\begin{array}{l}\text { 11-point } \\
\text { NRS, RMDQ }\end{array}$ \\
\hline $\begin{array}{l}\text { NCT00227292 } \\
\text { (withdrawn) }\end{array}$ & Chronic LBP & Germany & 2 & $\begin{array}{l}\text { Oral escitalopram } 10 \text { for } 1 \\
\text { week, } 20 \text { for } 3 \text { weeks }\end{array}$ & Placebo & 4 weeks & VAS, HDRS \\
\hline NCT01225068 & $\begin{array}{l}40 \text { participants with } \\
\text { chronic neuropathic LBP; } \\
\text { mean age } 47.7(10.3) \\
\text { years; } n=21(52 \%) \text { female }\end{array}$ & USA & 2 & $\begin{array}{l}\text { Oral milnacipran } 100, \\
\text { option to increase to } 200 \\
\text { after } 2 \text { weeks, } n=20 \text {. Drug } \\
\text { escalated in week } 1 \text { and } \\
\text { discontinued after week } 6\end{array}$ & Placebo, $n=20$ & 6 weeks & $\begin{array}{l}100 \mathrm{~mm} \\
\text { VAS }\end{array}$ \\
\hline $\begin{array}{l}\text { NCT03249558 } \\
\text { (ongoing) }\end{array}$ & $\begin{array}{l}\text { Chronic LBP or chronic } \\
\text { neck pain }\end{array}$ & USA & 3 & $\begin{array}{l}\text { Oral morphine } 60 \text { plus } \\
\text { duloxetine, or morphine } \\
\text { plus placebo duloxetine, } \\
\text { or placebo morphine plus } \\
\text { duloxetine } 60\end{array}$ & Placebo & 10 weeks & VAS \\
\hline $\begin{array}{l}\text { NCT03364075 } \\
\text { (crossover; } \\
\text { terminated) }\end{array}$ & Chronic LBP & NR & 3 & $\begin{array}{l}\text { Oral duloxetine } 30 \text { for } 1 \\
\text { week then } 60 \text { for } 1 \text { week } \\
\text { plus placebo, or } \\
\text { propranolol } 40 \text { for } 1 \text { week } \\
\text { then } 60 \text { for } 1 \text { week plus } \\
\text { placebo, or duloxetine } 30 \\
\text { for } 1 \text { week then } 60 \text { for } 1 \\
\text { week plus propranolol } 40 \\
\text { for } 1 \text { week then } 60 \text { for } 1 \\
\text { week }\end{array}$ & Placebo & $\begin{array}{l}2 \text { weeks/ } \\
\text { phase } \\
\text { with 1- } \\
\text { week } \\
\text { washout }\end{array}$ & Pain index \\
\hline $\begin{array}{l}\text { Pheasant } \\
\text { et al. [54] } \\
\text { (crossover) }\end{array}$ & $\begin{array}{l}16 \text { participants with } \\
\text { chronic LBP; mean age } \\
47.2 \text { years }{ }^{b} ; n=16(75 \%) \\
\text { female }\end{array}$ & USA & 2 & $\begin{array}{l}\text { Oral amitriptyline 50, } \\
n=6^{\mathrm{a}}\end{array}$ & $\begin{array}{l}\text { Active placebo } \\
\text { (atropine) } 0.2, n=10^{\mathrm{a}}\end{array}$ & $\begin{array}{l}6 \text { weeks/ } \\
\text { phase } \\
\text { with 2- } \\
\text { week } \\
\text { washout }\end{array}$ & $\begin{array}{l}\text { Functional } \\
\text { evaluation } \\
\text { rating }\end{array}$ \\
\hline $\begin{array}{l}\text { Schliessbach } \\
\text { et al. [55] } \\
\text { (crossover) }\end{array}$ & $\begin{array}{l}50 \text { participants with } \\
\text { chronic LBP; mean age } \\
54.4 \text { (17.3) years; } n=32 \\
(64 \%) \text { female }\end{array}$ & Switzerland & 2 & $\begin{array}{l}\text { Oral imipramine } 75 \text { single } \\
\text { dose, } n=50\end{array}$ & $\begin{array}{l}\text { Active placebo } \\
\text { (tolderodine) } 1.0 \text {, single } \\
\text { dose, } n=50\end{array}$ & $\begin{array}{l}2 \text { h/phase } \\
\text { with 1- } \\
\text { week } \\
\text { washout }\end{array}$ & $\begin{array}{l}\text { 11-point } \\
\text { NRS }\end{array}$ \\
\hline $\begin{array}{l}\text { Schukro et al. } \\
{[56]} \\
\text { (crossover) }\end{array}$ & $\begin{array}{l}41 \text { participants with } \\
\text { chronic LBP and leg pain; } \\
\text { mean age } 57.9 \text { years (13.4); } \\
n=21 \text { (51\%) female }\end{array}$ & Austria & 2 & $\begin{array}{l}\text { Oral duloxetine } 30 \text { to } 60 \\
\text { first week; } 60 \text { to } 120 \\
\text { second week; } 120 \text { for } 2 \\
\text { weeks, } n=16^{\mathrm{a}}\end{array}$ & Placebo, $n=18^{\mathrm{a}}$ & $\begin{array}{l}4 \text { weeks/ } \\
\text { phase } \\
\text { with 2- } \\
\text { week } \\
\text { washout }\end{array}$ & $\begin{array}{l}10 \mathrm{~cm} \text { VAS, } \\
\mathrm{BDI}, \mathrm{RMDQ}\end{array}$ \\
\hline $\begin{array}{l}\text { Skljarevski } \\
\text { et al. [57] }\end{array}$ & $\begin{array}{l}236 \text { participants with } \\
\text { chronic LBP, mean age } \\
\text { duloxetine groups } 51.8 \\
\text { (14.9) years; placebo } \\
\text { group } 51.2(13.5) \text { years; } \\
n=144(61 \%) \text { female }\end{array}$ & $\begin{array}{l}18 \text { clinical } \\
\text { sites in Brazil, } \\
\text { France, } \\
\text { Germany, } \\
\text { Mexico, and } \\
\text { Netherlands }\end{array}$ & 2 & $\begin{array}{l}\text { Oral duloxetine } 30 \text { for } 1 \\
\text { week, } 60 \text { for } 6 \text { weeks, non- } \\
\text { responders increased to } \\
120 / \text { day for remainder of } \\
\text { study, } n=115\end{array}$ & Placebo, $n=121$ & 13 weeks & $\begin{array}{l}\text { 11-point } \\
\text { NRS, BDI-II, } \\
\text { RMDQ }\end{array}$ \\
\hline $\begin{array}{l}\text { Skljarevski } \\
\text { et al. [58] }\end{array}$ & $\begin{array}{l}404 \text { participants with } \\
\text { chronic LBP; mean age } \\
\text { duloxetine } 20 \text { mg group }\end{array}$ & NR & 4 & $\begin{array}{l}\text { Oral duloxetine } 20, n=59 \text {, } \\
\text { or } 60, n=116 \text {, or } 120 \\
n=112\end{array}$ & Placebo, $n=117$ & 13 weeks & $\begin{array}{l}\text { 11-point } \\
\text { NRS, BDI-II, } \\
\text { RMDQ }\end{array}$ \\
\hline
\end{tabular}

52.9 (12.8) years,

duloxetine $60 \mathrm{mg}$ group

53.3 (14.7) years,

duloxetine $120 \mathrm{mg}$ group

54.9 (14.8) years, placebo

group 54 (13.5) years;

$n=232(57 \%)$ female 
Table 1 Characteristics of included studies (Continued)

\begin{tabular}{|c|c|c|c|c|c|c|c|}
\hline $\begin{array}{l}\text { Study } \\
\text { Label, } \\
\text { citation }\end{array}$ & $\begin{array}{l}\text { Patient sample } \\
\text { Study level data unless } \\
\text { reported otherwise; } \\
\text { mean (SD) }\end{array}$ & Setting & $\begin{array}{l}\text { Number } \\
\text { of trial } \\
\text { arms }\end{array}$ & $\begin{array}{l}\text { Intervention, number } \\
\text { assigned } \\
\text { (mg/day unless } \\
\text { indicated) }\end{array}$ & $\begin{array}{l}\text { Comparator, number } \\
\text { assigned } \\
\text { (mg/day unless } \\
\text { indicated) }\end{array}$ & $\begin{array}{l}\text { Duration } \\
\text { of } \\
\text { treatment }\end{array}$ & $\begin{array}{l}\text { Outcome } \\
\text { measures } \\
\text { applicable } \\
\text { to this } \\
\text { review }\end{array}$ \\
\hline $\begin{array}{l}\text { Skljarevski } \\
\text { et al. [59] }\end{array}$ & $\begin{array}{l}401 \text { participants with } \\
\text { chronic LBP, mean age } \\
\text { duloxetine group } 54.9 \\
\text { (13.7) years; placebo } \\
\text { group } 53.4(14.2) \text { years; } \\
n=246(61 \%) \text { female }\end{array}$ & $\begin{array}{l}27 \text { sites in } \\
\text { Germany, } \\
\text { Netherlands, } \\
\text { Poland, } \\
\text { Russia, Spain, } \\
\text { and USA }\end{array}$ & 2 & $\begin{array}{l}\text { Oral duloxetine 60, } \\
n=198\end{array}$ & Placebo, $n=203$ & 12 weeks & $\begin{array}{l}\text { 11-point } \\
\text { NRS, BDI-II, } \\
\text { RMDQ }\end{array}$ \\
\hline $\begin{array}{l}\text { Treves et al. } \\
{[60]}\end{array}$ & $\begin{array}{l}68 \text { participants with } \\
\text { chronic LBP, LBP with leg } \\
\text { pain, and sciatica; mean } \\
\text { age } 45.6^{\text {b }} \text { years; } n=35 \\
(51 \%) \text { female }\end{array}$ & France & 3 & $\begin{array}{l}\text { IV clomipramine, } \\
\text { progressive doses in the } \\
\text { morning until maximum } \\
\text { dose of } 75 \text { reached ( } 3 r d \\
\text { day) and maintained for } \\
\text { the next } 7 \text { days with } \\
\text { placebo in evening, } \\
n=25 \text {, or IV clomipramine } \\
\text { progressive doses in the } \\
\text { evening until maximum } \\
\text { dose of } 75 \text { reached ( } 3 r d \\
\text { day) and maintained for } \\
\text { the next } 7 \text { days with } \\
\text { placebo in morning, } \\
n=27\end{array}$ & $\begin{array}{l}\text { IV placebo (isotonic } \\
\text { glucose), } n=16\end{array}$ & 10 days & $10 \mathrm{~cm}$ VAS \\
\hline $\begin{array}{l}\text { Urquhart } \\
\text { et al. [61] }\end{array}$ & $\begin{array}{l}146 \text { participants with } \\
\text { chronic LBP; mean age } \\
\text { amitriptyline group } 53.5 \\
\text { (14.2) years, placebo } \\
\text { group } 56.0(13.2) \text { years; } \\
n=56(38 . \%) \text { female }\end{array}$ & Australia & 2 & $\begin{array}{l}\text { Oral amitriptyline } 25, \\
n=72\end{array}$ & $\begin{array}{l}\text { Active placebo } \\
\text { (benztropine mesylate) } \\
1.0, n=74\end{array}$ & 6 months & $\begin{array}{l}100 \mathrm{~mm} \\
\text { VAS, BDI, } \\
\text { RMDQ }\end{array}$ \\
\hline
\end{tabular}

NR not reported, LBP low back pain, SBPQ Short Back Pain Questionnaire, BDI Beck Depression Inventory, BDI-II Beck Depression Inventory II, DDS Descriptor Differential Scale, RMDQ Roland Morris Disability Questionnaire, VAS visual analog scale, MADRS Montgomery Asberg Depression Rating Scale, NRS numerical rating scale, BPI Brief Pain Inventory, HDRS Hamilton Depression Rating Scale

${ }^{a}$ Number of participants randomized in the first phase of crossover trial

${ }^{b}$ No measure of central tendency reported

${ }^{\mathrm{C}} \mathrm{Age}$ and sex data presented for intention-to-treat sample

(Fig. 1). We excluded 63 records and included 60 records that comprised 23 unique trials [51-69] (Table 1).

Eighteen trials used a parallel design, and five trials used crossover designs. Four trials were reported in trial registries. We identified a single ongoing trial, a single withdrawn trial, and a single terminated trial. Seventeen trials provided data for inclusion in the meta-analysis. These 17 trials randomized a total 2517 participants to one or more of 11 different antidepressant medicines or placebo. We did not identify any trials of antidepressant medicines compared to waiting list, usual care or notreatment. The analyses presented below are for the effect of antidepressant medicines compared to placebo.

\section{Risk of bias}

We assessed completed trials $(n=20)$ for overall risk of bias (Table S1 in Additional file 2); 15 were assessed as high risk, four at moderate risk, and a single trial at low risk of bias. All twenty trials reported an appropriate method of blinding. Fourteen trials reported either high dropout rates or differences in dropouts between arms.
Seven trials reported that they maintained complete control over the publication of results or had no fundingrelated conflicts of interests.

\section{Assessment of publication bias}

Visual inspection of funnel plots for each outcome suggested that the effects were evenly distributed around the mean (Figures S1-14 in Additional file 2). For all outcomes, visual inspection of contour-enhanced funnel plots provided no evidence of effects clustered around the threshold for statistical significance. Egger's tests were conducted for outcomes with 10 studies; only a single study indicated statistically significant asymmetry. A single completed trial report from a trial registry (NCT01225068) was included in our analyses.

\section{Confidence in evidence}

The GRADE assessment of confidence in the evidence for each main analysis is presented in Appendix S2 in Additional file 2 and referred to below. 


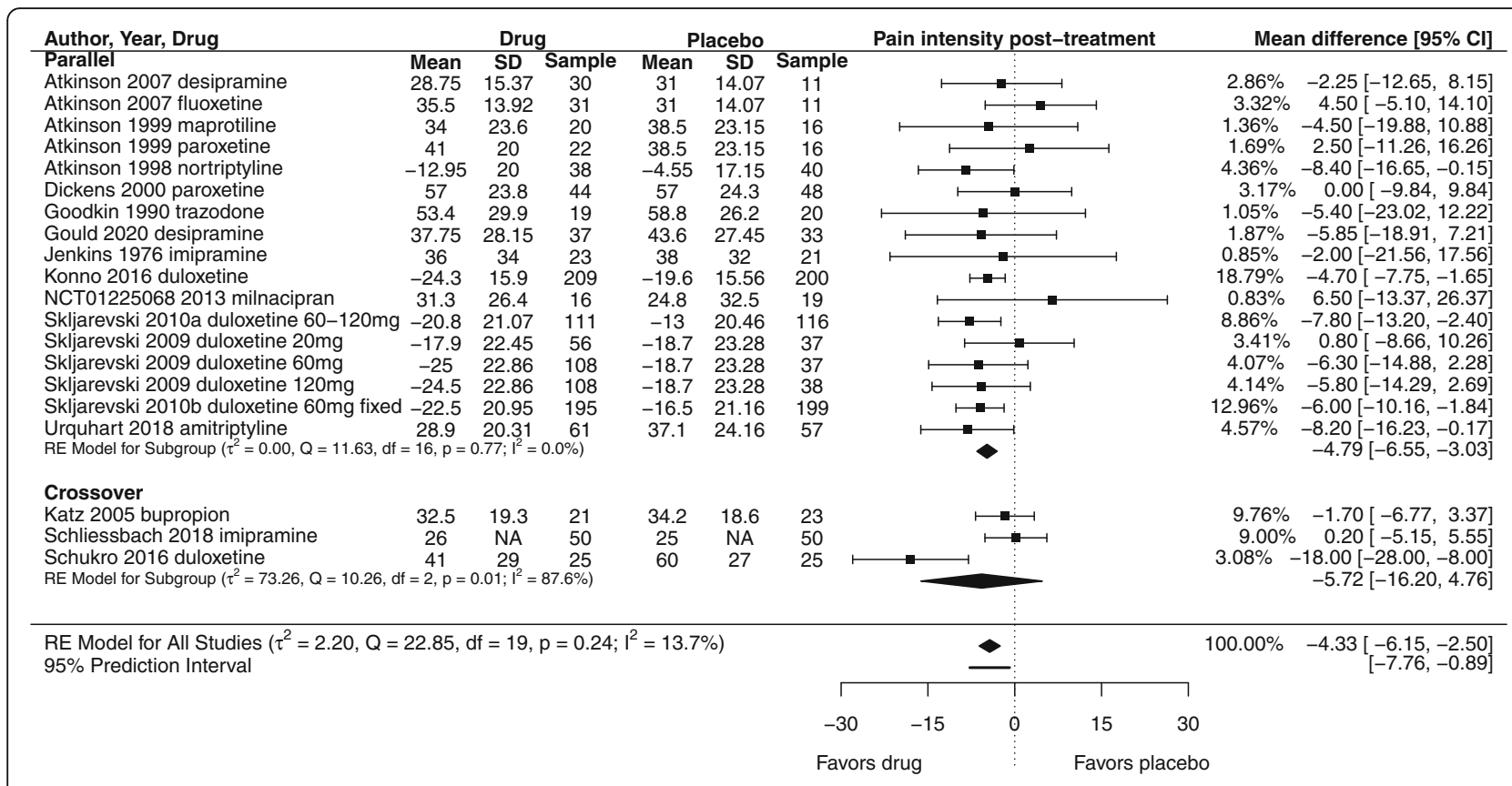

Fig. 2 Effect of antidepressants compared to placebo on pain intensity (0-100 scale) for patients with LBP. Negative values for mean outcomes indicate change from baseline. Negative values for mean difference indicate effect favors drug compared to placebo. NA= group SD data not available; between-group summary statistics used in meta-analysis

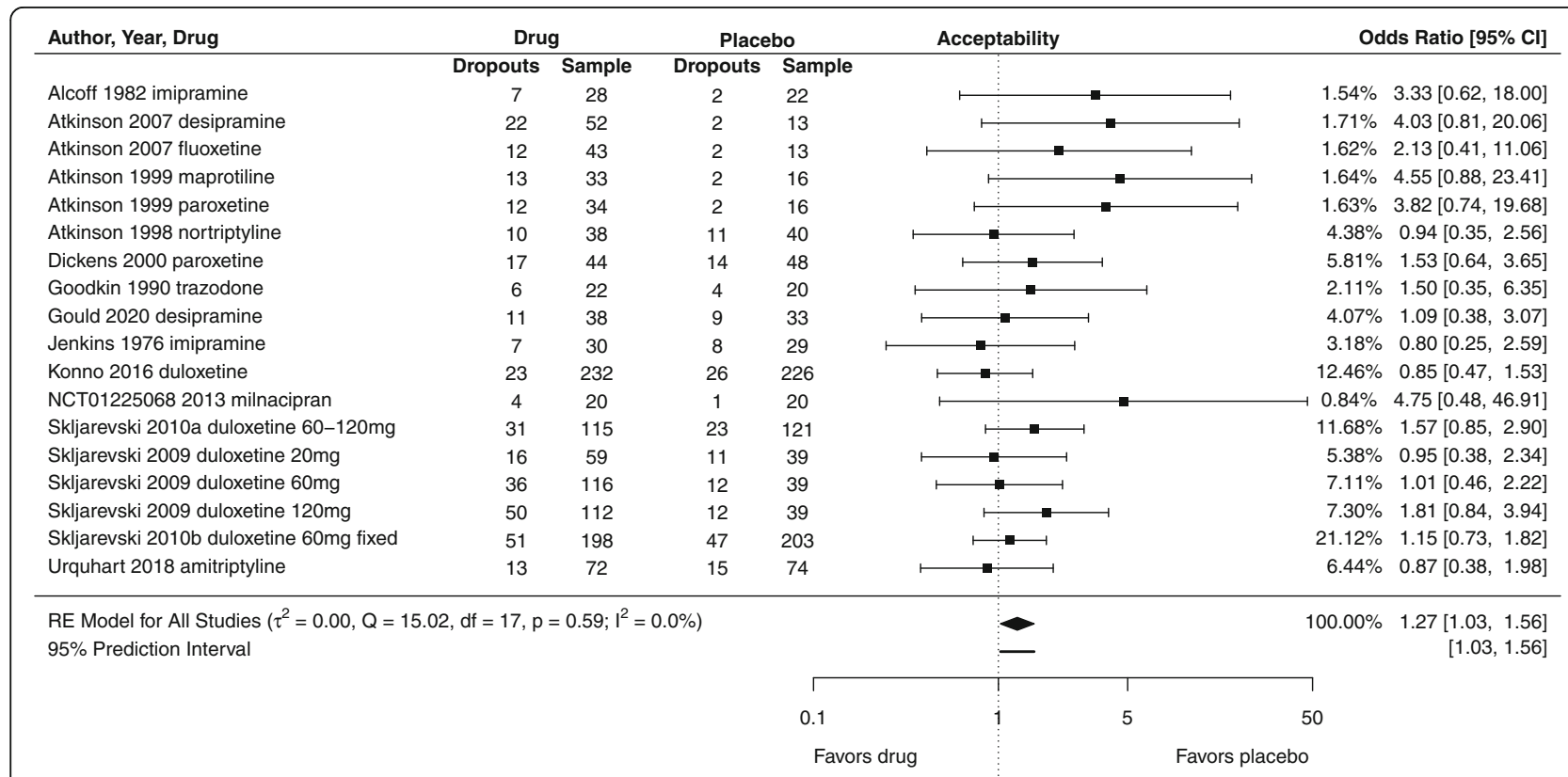

Fig. 3 All-cause discontinuation (acceptability) of antidepressants compared to placebo for patients with LBP. Odds ratio greater than 1 indicates greater odds of discontinuation in antidepressant group (i.e., effect favors placebo) 


\section{Main analysis}

\section{Primary outcome: pain}

Sixteen of the 23 included trials reported data for pain. We downgraded confidence in the evidence by two levels due to trial limitations. There is low confidence that the pooled effect of antidepressant medicines compared to placebo is - 4.33 [95\% CI - 6.15 to - 2.50; $\left.\mathrm{Tau}^{2}=2.20\right]$ on a $0-100$ point scale (Fig. 2).

\section{Primary outcome: acceptability}

Fourteen of the 23 included trials reported data for acceptability (all-cause discontinuation). We downgraded confidence in the evidence by two levels due to trial limitations. There is low confidence that the odds of allcause discontinuation are higher for antidepressants than for placebo: odds ratio 1.27 [95\% CI 1.03 to 1.56; $\left.\mathrm{Tau}^{2}=0\right]$ (Fig. 3).

\section{Secondary outcome: function}

Six of the 23 included trials reported data for function. We downgraded confidence in the evidence by two levels due to trial limitations. There is low confidence that the pooled effect of antidepressants compared to placebo is -3.22 [95\% CI -4.96 to $\left.-1.48, \mathrm{Tau}^{2}=0\right]$ on a 0-100 point scale (Figure S15 in Additional file 2).

\section{Secondary outcome: symptoms of depression}

Four of the 23 included trials reported data for symptoms of depression. We downgraded confidence in the evidence by two levels for trial limitations and an additional level for imprecision. There is very low confidence that the pooled effect of antidepressants compared to placebo is -1.72 [95\% CI -3.88 to $0.44 ; \mathrm{Tau}^{2}=0$ ] (Figure S16 in Additional file 2) on a 0-100 point scale.

\section{Secondary outcome: safety}

Nine of the 23 included trials reported data for safety (adverse effects). We downgraded confidence in the evidence by two levels for trial limitations. There is low confidence that the odds of experiencing an adverse effect are higher for antidepressants than for placebo: odds ratio 1.58 [95\% CI 1.28 to 1.93 ; $\mathrm{Tau}^{2}=0$ ] (Figure S17 in Additional file 2).

\section{Secondary outcome: harm}

Six of the 23 included trials reported data for harm (serious adverse effects). We downgraded confidence in the evidence by two levels for trial limitations and an additional level for imprecision. There is very low confidence that the odds of experiencing a serious adverse effect are higher for antidepressants than for placebo: odds ratio 1.29 [95\% CI 0.56 to 2.94; $\mathrm{Tau}^{2}=0$ ] (Figure S18 in Additional file 2).

\section{Secondary outcome: tolerability}

Ten of the 23 included trials reported data for tolerability (discontinuation due to adverse effects). We downgraded confidence in the evidence by two levels for trial limitations. There is low confidence that the

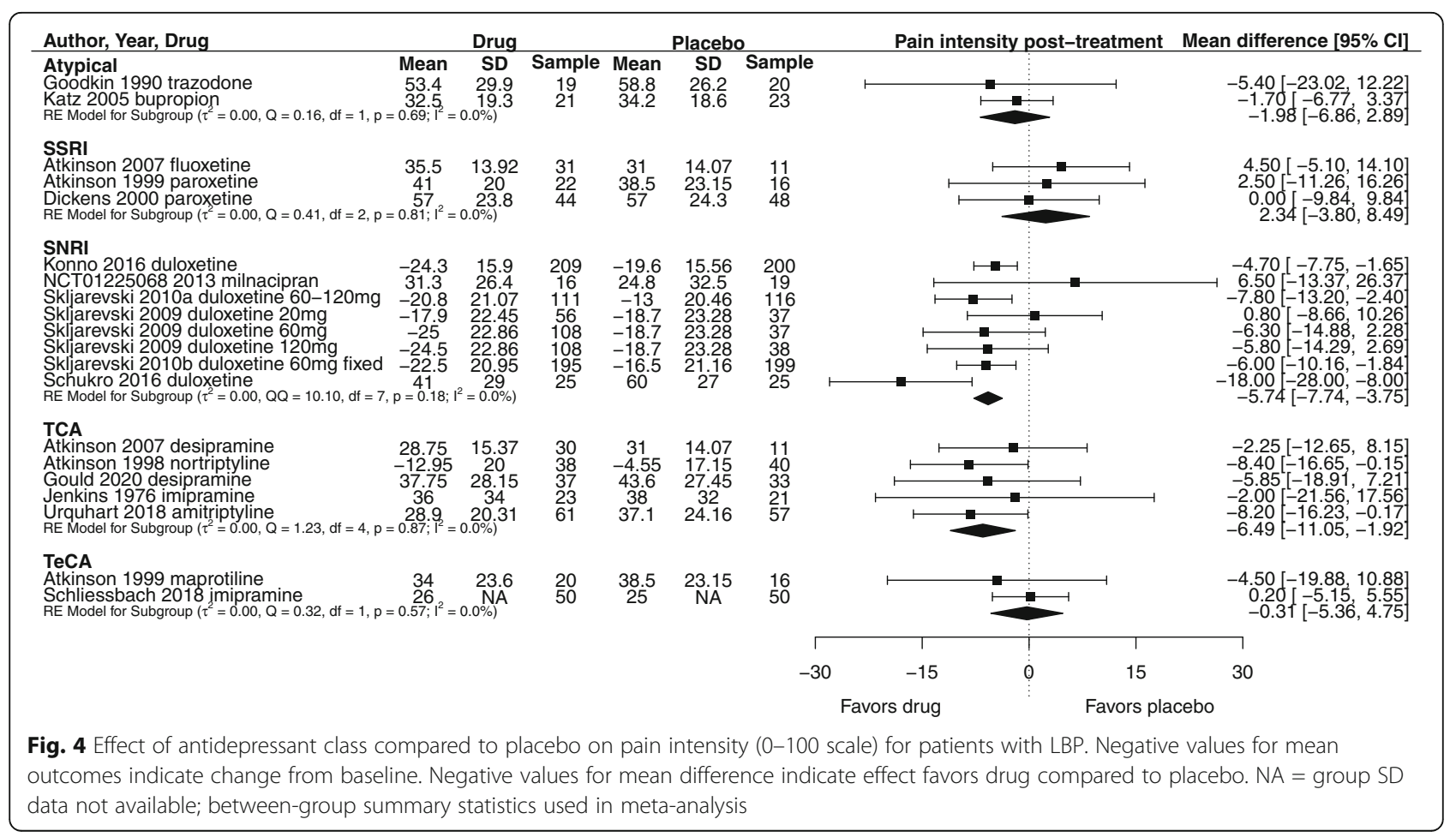


odds of discontinuing treatment due to an adverse effect are higher for antidepressants than for placebo: odds ratio 2.39 [95\% CI 1.71 to 3.34; $\mathrm{Tau}^{2}=0$ ] (Figure S19 in Additional file 2).

\section{Other analyses}

\section{Subgroup analyses}

We conducted subgroup analyses for pain by antidepressant type and dose to provide additional clinical information (Fig. 4). There were no trials that evaluated the efficacy of HCA or MAOI antidepressants on LBP symptoms. The results for additional subgroup and sensitivity analyses are presented in Supplementary results with corresponding forest plots in Figures S20-23 in Additional file 2 .

\section{Influence of further research on results}

The extended funnel plots (Figures S24, S25 in Additional file 2) suggest the upper bound of the confidence interval for the pooled effect would cross the threshold for clinical meaningfulness if the meta-analysis included an additional hypothetical trial with approximately 400 participants per arm and an effect for pain of approximately -30 on a $0-100$ scale (antidepressants more favorable than placebo).

\section{Post hoc effects of duloxetine}

Duloxetine is noted in the 2017 American College of Physicians guideline to have small effects on pain and function compared to placebo, for chronic LBP [11]. We repeated the main analyses on five trials that evaluated duloxetine compared to placebo. The effect of duloxetine on pain intensity post-treatment was -5.87 [95\% CI - 7.88 to - 3.86; $\mathrm{Tau}^{2}=0$ ) (Figure S26 in Additional file 2 ). The odds ratio for all-cause discontinuation of duloxetine compared to placebo was 1.17 [95\% CI 0.90 to 1.52; $\mathrm{Tau}^{2}=0$ ] (Figure S27 in Additional file 2). The odds ratio for experiencing adverse effects of duloxetine compared to placebo was 1.50 [95\% CI 1.21 to 1.85 ; $\mathrm{Tau}^{2}=0$ ] (Figure S28 in Additional file 2). The odds ratio for experiencing serious adverse effects of duloxetine compared to placebo was 1.35 [95\% CI 0.56 to 3.27; $\mathrm{Tau}^{2}=0$ ] (Figure S29 in Additional file 2). The odds ratio for discontinuing treatment due to adverse effects of duloxetine compared to placebo was 2.53 [95\% CI 1.70 to 3.77; $\left.\mathrm{Tau}^{2}=0\right]$ (Figure S30 in Additional file 2).

\section{Post hoc sensitivity analyses}

The REML estimator may underestimate between-study variance for binary outcomes when events are rare [70]. We repeated the analyses for acceptability, safety, harm, and tolerability using DerSimonian-Laird, Paule and Mandel and Mantel-Haenszel methods of estimation (Table S2 in Additional file 2). A single additional post hoc sensitivity analysis is reported in Supplementary Results and Figure S31 in Additional file 2.

\section{Discussion}

We conducted a systematic review to evaluate the effect of antidepressant medicines for patients with LBP. We included 23 trials in the systematic review and up to 17 in the meta-analyses. There is low confidence in evidence that, on average, patients with LBP treated with antidepressant medicines will experience a small improvement in pain and function and no improvement in symptoms of depression, compared to placebo. These effects are not clinically important $[42,71]$. There is low confidence in evidence that patients are at increased odds of experiencing an adverse or serious adverse effect and at increased odds of stopping treatment due to an adverse effect or another reason, compared to placebo. Taken together, these data indicate treatment of LBP symptoms with antidepressants has no important benefit; is less acceptable, less safe and less tolerable; and may be harmful, compared to treatment with a placebo medicine.

A recent overview of clinical guidelines reported that 6 of 8 international guidelines recommend the use of antidepressants for chronic LBP where necessary [72]. The current American College of Physicians clinical guideline for the management of LBP [11] recommends the use of duloxetine for chronic LBP as second-line therapy where non-pharmacological therapy has been unsuccessful. This might be reconsidered in view of our findings. The analyses of duloxetine specifically showed a small effect on pain that is unlikely clinically important [73] and higher odds of adverse effects and dropout due to adverse effects compared to placebo.

Our work has a number of strengths. We adhered to a prospectively registered protocol and reported findings in line with recommendations [74]. Our searches are extensive and up to date and we included data from trial registry reports $[29,75,76]$. We also evaluated the acceptability, safety, harm, and tolerability of antidepressant medicines, in addition to effects on symptoms. This addresses limitations of the most recent review, which included 11 fewer trials and did not evaluate adverse effects [23]. The observed low heterogeneity across all outcomes, together with the improved precision of the estimates, substantiates our findings and interpretation. We determined that different methods of estimation did not influence these observations and note that similar homogeneity for binary outcomes has been reported in other large meta-analyses for antidepressant medicines [15]. We estimated parameters for a hypothetical future trial that would meaningfully impact the effect estimate for pain, to assist readers' interpretation of the need for further trials.

We were unable to estimate effects for the long-term efficacy and acceptability of antidepressants because 
such data were reported in a single trial [61]. We were also unable to evaluate the effects of antidepressants in patients with acute LBP because we identified no usable data. The hypothetical future trial parameters estimated with the extended funnel plot do not consider risk of bias and are not estimable for binary outcomes.

\section{Conclusion}

This review demonstrates that treatment of LBP symptoms with antidepressants has no important benefit; is less acceptable, less safe, and less tolerable; and may be harmful, compared to treatment with a placebo medicine. This evidence is supported by homogenous, precise effect sizes across outcomes. These findings provide Level I evidence to guide clinicians in their use of antidepressants to treat LBP.

\section{Abbreviations}

BDI: Beck Depression Inventory; BDI-II: Beck Depression Inventory II; BPI: Brief Pain Inventory; DDS: Descriptor Differential Scale; GRADE: Grading of Recommendations Assessment Development and Evaluation; HCA: Heterocyclic antidepressant; HDRS: Hamilton Depression Rating Scale; LBP: Low back pain; MADRS: Montgomery Asberg Depression Rating Scale; MAOI: Monoamine oxidase inhibitor; NRS: Numerical rating scale; NSAI D: Non-steroidal anti-inflammatory; PRISMA: Preferred Reporting Items for Systematic Reviews and Meta-Analyses; RCT: Randomized controlled trial; REML: Restricted maximum likelihood; RMDQ: Roland Morris Disability Questionnaire; SBPQ: Short Back Pain Questionnaire; SNRI: Serotonin and norepinephrine reuptake inhibitor; SSRI: Selective serotonin reuptake inhibitor; TCA: Tricyclic antidepressant; TeCA: Tetracyclic antidepressant; VAS: Visual analog scale

\section{Acknowledgements}

No acknowledgements.

\section{Authors' contributions}

MCF had full access to all of the data in the study and takes full responsibility for the integrity of the data and the accuracy of the data analysis. JHM and MKB conceived the study idea and designed the study; MCF, MKB, and MAW created the search terms and conducted the database searches; MCF, MAW, AGC, HBL, RRNR, and MDJ extracted the data; MCF and MAW analyzed the data; MCF drafted and revised the manuscript; MKB, JHM, $\mathrm{CKL}, \mathrm{RD}$, and SMG made substantial contributions to the interpretation of results and critical revision of the manuscript. All authors approved the final version of the manuscript.

\section{Funding}

No external funding was received for this study. MCF is supported by an Australian Medical Research Future Fund Grant GNTID1170205. MKB is supported by a NeuRA PhD Candidature Scholarship and Supplementary Scholarship and was additionally funded during this work by an Australian Government Research Training Program Scholarship and a UNSW Research Excellence Award. MAW is supported by a University Postgraduate Award and School of Medical Sciences Top-Up Scholarship from the University of New South Wales, and a Postgraduate Scholarship from the National Health and Medical Research Council of Australia. AGC is supported by the University of New South Wales Prince of Wales Clinical School Postgraduate Research Scholarship and a NeuRA PhD Candidature Supplementary Scholarship. HBL is supported by Australian Government post-graduate award. RRNR is supported by the University of New South Wales School of Medical Sciences Postgraduate Research Scholarship and a NeuRA PhD Candidature Supplementary Scholarship. The funders/sponsors had no role in the design and conduct of the study; collection, management, analysis, and interpretation of the data; preparation, review, or approval of the manuscript; and decision to submit the manuscript for publication.

\section{Availability of data and materials}

The dataset used and analyzed during the current study are available from the corresponding author on reasonable request.

Ethics approval and consent to participate

Not applicable.

\section{Consent for publication}

Not applicable.

\section{Competing interests}

The authors declare no competing interests.

\section{Author details}

${ }^{1}$ Centre for Pain IMPACT, Neuroscience Research Australia, Sydney, NSW, Australia. ${ }^{2}$ School of Health Sciences, University of New South Wales, Sydney, Australia. ${ }^{3}$ Prince of Wales Clinical School, University of New South Wales, Sydney, Australia. ${ }^{4} \mathrm{New}$ College Village, University of New South Wales, Sydney, Australia. ${ }^{5}$ IMPACT in Health, University of South Australia, Adelaide, Australia. ${ }^{6}$ School of Psychology, University of New South Wales, Sydney, Australia. ${ }^{7}$ Clinical Pharmacology \& Toxicology, St. Vincent's Hospital, Sydney, Australia. ${ }^{8}$ St. Vincent's Clinical School, Faculty of Medicine, University of New South Wales, Sydney, Australia. ${ }^{9}$ School of Psychiatry, University of New South Wales, Sydney, Australia. ${ }^{10}$ Black Dog Institute, Sydney, Australia.

Received: 28 October 2020 Accepted: 26 January 2021

Published online: 24 February 2021

\section{Supplementary Information}

The online version contains supplementary material available at https://doi. org/10.1186/s13643-021-01599-4.

Additional file 1. PRISMA 2009 Checklist.

Additional file 2. Supplementary Content.

\section{Author details}

The online version contains supplementary material available at https://doi. org/10.1186/s13643-021-01599-4.

${ }^{1}$ Centre for Pain IMPACT, Neuroscience Research Australia, Sydney, NSW, Australia. ${ }^{2}$ School of Health Sciences, University of New South Wales, Sydney, Australia. ${ }^{3}$ Prince of Wales Clinical School, University of New South Wales, Sydney, Australia. ${ }^{4}$ New College Village, University of New South Wales, Sydney, Australia. ${ }^{5}$ IIMPACT in Health, University of South Australia, Adelaide, Australia. ${ }^{6}$ School of Psychology, University of New South Wales, Sydney, Australia. ${ }^{7}$ Clinical Pharmacology \& Toxicology, St. Vincent's Hospital, Sydney, Australia. ${ }^{8}$ St. Vincent's Clinical School, Faculty of Medicine, University of New South Wales, Sydney, Australia. ${ }^{9}$ School of Psychiatry, University of New South Wales, Sydney, Australia. ${ }^{10}$ Black Dog Institute, Sydney, Australia.

Received: 28 October 2020 Accepted: 26 January 2021

Published online: 24 February 2021

\section{References}

1. James SL, Abate D, Abate KH, Abay SM, Abbafati C, Abbasi N, et al. Global, regional, and national incidence, prevalence, and years lived with disability for 354 diseases and injuries for 195 countries and territories, 1990-2017: a systematic analysis for the Global Burden of Disease Study 2017. Lancet. 2018;392(10159):1789-858.

2. Gore M, Tai KS, Sadosky A, Leslie D, Stacey BR. Use and costs of prescription medications and alternative treatments in patients with osteoarthritis and chronic low back pain in community-based settings. Pain Pract. 2012;12(7): 550-60.

3. Hart OR, Uden RM, McMullan JE, Ritchie MS, Williams TD, Smith BH. A study of National Health Service management of chronic osteoarthritis and low back pain. Prim Health Care Res Dev. 2015;16(02):157-66.

4. Piccoliori G, Engl A, Gatterer D, Sessa E, in der Schmitten J, Abholz H-H. Management of low back pain in general practice - is it of acceptable quality: an observational study among 25 general practices in South Tyrol (Italy). BMC Fam Pract. 2013;14(1):148. 
5. Ivanova Jl, Birnbaum HG, Schiller M, Kantor E, Johnstone BM, Swindle RW. Real-world practice patterns, health-care utilization, and costs in patients with low back pain: the long road to guideline-concordant care. Spine J. 2011;11(7):622-32.

6. Williams CM, Maher CG, Hancock MJ, McAuley JH, McLachlan AJ, Britt H, et al. Low back pain and best practice care. Arch Intern Med. 2010;170(3):271.

7. Bishop P, Wing P. Compliance with clinical practice guidelines in family physicians managing worker's compensation board patients with acute lower back pain. Spine J. 2003;3(6):442-50.

8. Almeida M, Saragiotto B, Richards B, Maher CG. Primary care management of non-specific low back pain: key messages from recent clinical guidelines. Med J Aust. 2018;208(6):272-5.

9. National Institute for Health and Care Excellence (NICE) 2016. Low back pain and sciatica in over $16 \mathrm{~s}$ : assessment and management (NG59). https:// www.nice.org.uk/guidance/ng59. Accessed 20 February 2020.

10. Wambeke P, Desomer A, Ailliet L, Berquin A, Demoulin C, Depreitere B, et al. Low back pain and radicular pain: assessment and management. Belgian Health Care Knowledge Centre (KCE). 2017.

11. Qaseem A, Wilt TJ, McLean RM, Forciea MA. Noninvasive treatments for acute, subacute, and chronic low back pain: a clinical practice guideline from the American college of physicians. Ann Intern Med. 2017;166(7):514.

12. Shmagel A, Ngo L, Ensrud K, Foley R. Prescription medication use among community-based U.S. adults with chronic low back pain: a cross-sectional population based study. J Pain. 2018;19(10):1104-12.

13. Thackeray A, Hess R, Dorius J, Brodke D, Fritz J. Relationship of opioid prescriptions to physical therapy referral and participation for medicaid patients with new-onset low back pain. J Am Board Fam Med. 2017;30(6):784-94.

14. Mathieson S, Valenti L, Maher CG, Britt H, Li Q, McLachlan AJ, et al. Worsening trends in analgesics recommended for spinal pain in primary care. Eur Spine J. 2018;27(5):1136-45.

15. Cipriani A, Furukawa TA, Salanti G, Chaimani A, Atkinson LZ, Ogawa Y, et al. Comparative efficacy and acceptability of 21 antidepressant drugs for the acute treatment of adults with major depressive disorder: a systematic review and network meta-analysis. Lancet. 2018:391(10128):1357-66.

16. Harmer CJ, Duman RS, Cowen PJ. How do antidepressants work? New perspectives for refining future treatment approaches. The Lancet Psychiatry. 2017;4(5):409-18.

17. Cohen SP, Abdi S. New developments in the use of tricyclic antidepressants for the management of pain. Curr Opin Anaesthesiol. 2001;14(5):505-11.

18. Koes BW, Backes D, Bindels PJE. Pharmacotherapy for chronic non-specific low back pain: current and future options. Expert Opin Pharmacother. 2018; 19(6):537-45.

19. Mafi JN, McCarthy EP, Davis RB, Landon BE. Worsening trends in the management and treatment of back pain. JAMA Intern Med. 2013;173(17):1573.

20. Salerno SM, Browning $R$, Jackson JL. The effect of antidepressant treatment on chronic back pain. Arch Intern Med. 2002;162(1):19.

21. Staiger TO, Gaster B, Sullivan MD, A Deyo R. Systematic review of antidepressants in the treatment of chronic low back pain. Spine. 2003; 28(22):2540-5.

22. Schnitzer TJ, Ferraro A, Hunsche E, Kong SX. A comprehensive review of clinical trials on the efficacy and safety of drugs for the treatment of low back pain. J Pain Symptom Manage. 2004;28(1):72-95.

23. Urquhart DM, Hoving JL, Assendelft WJ, Roland M, van Tulder MW. Antidepressants for non-specific low back pain. Cochrane Database Syst Rev. 2008:44(8):085201.

24. Ferraro MC, Bagg MK, Folly de Campos T, McAuley JH. RADICAL: Systematic review of anti-depressant medicines if considered analgesics for low back pain. 2019. https://osf.io/6gurb/

25. Moher D, Liberati A, Tetzlaff J, Altman DG. Preferred reporting items for systematic reviews and meta-analyses: the PRISMA statement. BMJ. 2009; 339:b2535.

26. Cipriani A, Furukawa TA, Salanti G, Geddes JR, Higgins JP, Churchill R, et al. Comparative efficacy and acceptability of 12 new-generation antidepressants: a multiple-treatments meta-analysis. Lancet. 2009;373(9665): 746-58

27. United States Food and Drugs Administration. Code of Federal Regulations Title 21. 2018. https://www.accessdata.fda.gov/scripts/cdrh/cfdocs/cfcfr/ cfrsearch.cfm?fr = 314.80. Accessed 31 July 2019

28. Bagg MK, McLachlan AJ, Maher CG, Kamper SJ, Williams CM, Henschke N, et al. Paracetamol, NSAIDS and opioid analgesics for chronic low back pain: a network meta-analysis. Cochrane Database Syst Rev. 2018(6):CD013045.
29. Bagg MK, O'Hagan E, Zahara P, Wand BM, Hübscher M, Moseley GL, et al. Systematic reviews that include only published data may overestimate the effectiveness of analgesic medicines for low back pain: a systematic review and meta-analysis. J Clin Epidemiol. 2020;124:149-159.

30. Dionne CE, Dunn KM, Croft PR, Nachemson AL, Buchbinder R, Walker BF, et al. A consensus approach toward the standardization of back pain definitions for use in prevalence studies. Spine. 2008;33(1):95-103.

31. Koes BW, van Tulder MW, Peul WC. Diagnosis and treatment of sciatica. BMJ. 2007:334(7607):1313-7.

32. WHO Collaborating Centre for Drug Statistics Methodology. ATC/DDD Index. 2020. https://www.whocc.no/atc_ddd_index/. Accessed 31 July 2019

33. U.S. Food and Drug Administration. Drugs@FDA. 2020. https://www.fda.gov/ drugs. Accessed 31 July 2019

34. Australian Government Department of Health Therapeutic Goods Administration. Australian Register of Therapeutic Goods. 2020. https:// www.tga.gov.au/searching-australian-register-therapeutic-goods-artg. Accessed 31 July 2019

35. Medicines and Healthcare Products Regulatory Agency. MHRA Products. 2020 https://products.mhra.gov.uk. Accessed 31 July 2019

36. European Medicines Agency. Medicines. 2020. https://www.ema.europa.eu/ en/medicines. Accessed 31 July 2019

37. Higgins J, Green S. Cochrane handbook for systematic reviews of interventions version 5.1.0 (updated March 2011). Cochrane Collaboration; 2011.

38. Furlan AD, Malmivaara A, Chou R, Maher CG, Deyo RA, Schoene M, et al. 2015 updated method guideline for systematic reviews in the cochrane back and neck group. Spine. 2015;40(21):1660-73.

39. Furukawa TA, Salanti G, Atkinson LZ, Leucht S, Ruhe HG, Turner EH, et al. Comparative efficacy and acceptability of first-generation and secondgeneration antidepressants in the acute treatment of major depression: protocol for a network meta-analysis. BMJ Open. 2016;6(7):e010919.

40. Wan X, Wang W, Liu J, Tong T. Estimating the sample mean and standard deviation from the sample size, median, range and/or interquartile range. BMC Med Res Methodol. 2014;14(1):135

41. Higgins JPT, Thomas J, Chandler J, Cumpston M, Li T, Page MJ, et al., Cochrane Handbook for Systematic Reviews of Interventions Version 6.0. Wiley; 2019.

42. Busse JW, Bartlett SJ, Dougados M, Johnston BC, Guyatt GH, Kirwan JR, et al. Optimal strategies for reporting pain in clinical trials and systematic reviews: recommendations from an OMERACT 12 workshop. J Rheumatol. 2015; 42(10):1962-70

43. R Core Team. R: a language and environment for statistical computing. Vienna, Austria: R Foundation for Statistical Computing; 2019. https://www.rproject.org/

44. Viechtbauer W. Conducting meta-analyses in $\mathrm{R}$ with the metafor package. J Stat Softw. 2010;36(3):1-48.

45. PDR LLC. Prescriber's digital reference. 2020. https://www.pdr.net. Accessed 24 February 2020

46. StataCorp LLC. Stata Statistical Software. College Station, TX; 2019.

47. Ferreira ML, Herbert RD, Crowther MJ, Verhagen A, Sutton AJ. When is a further clinical trial justified? BMJ. 2012;345:e5913.

48. Langan D, Higgins JPT, Gregory W, Sutton AJ. Graphical augmentations to the funnel plot assess the impact of additional evidence on a meta-analysis. J Clin Epidemiol. 2012;65(5):511-9.

49. Guyatt GH, Oxman AD, Vist GE, Kunz R, Falck-Ytter Y, Alonso-Coello P, et al. GRADE: an emerging consensus on rating quality of evidence and strength of recommendations. BMJ. 2008;336(7650):924-6.

50. Sterne JAC, Sutton AJ, Ioannidis JPA, Terrin N, Jones DR, Lau J, et al. Recommendations for examining and interpreting funnel plot asymmetry in meta-analyses of randomised controlled trials. BMJ. 2011; 343:d4002.

51. Alcoff J, Jones E, Rust P, Newman R. Controlled trial of imipramine for chronic low back pain. J Fam Pract. 1982;14(5):841-6.

52. Atkinson JH, Slater MA, Capparelli EV, Wallace MS, Zisook S, Abramson I, et al. Efficacy of noradrenergic and serotonergic antidepressants in chronic back pain. J Clin Psychopharmacol. 2007;27(2):135-42.

53. Konno S, Oda N, Ochiai T, Alev L. Randomized, double-blind, placebocontrolled phase III trial of duloxetine monotherapy in Japanese patients with chronic low back pain. Spine. 2016;41(22):1709-17.

54. Pheasant H, Bursk A, Goldfarb J, Azen SP, Weiss JN, Borelli L. Amitriptyline and chronic low-back pain: a randomized double-blind crossover study. Spine. 1983;8(5):552-7. 
55. Schliessbach J, Siegenthaler A, Bütikofer L, Limacher A, Juni P, Vuilleumier PH et al. Effect of single-dose imipramine on chronic low-back and experimental pain. A randomized controlled trial. PLoS One. 2018;13(5):e0195776.

56. Schukro RP, Oehmke MJ, Geroldinger A, Heinze G, Kress H-G, Pramhas S. Efficacy of duloxetine in chronic low back pain with a neuropathic component. Anesthesiology. 2016;124(1):150-8.

57. Skljarevski V, Desaiah D, Liu-Seifert H, Zhang Q, Chappell AS, Detke MJ, et al. Efficacy and safety of duloxetine in patients with chronic low back pain. Spine. 2010;35(13):E578-85.

58. Skljarevski V, Ossanna M, Liu-Seifert H, Zhang Q, Chappell A, lyengar S, et al. A double-blind, randomized trial of duloxetine versus placebo in the management of chronic low back pain. Eur J Neurol. 2009;16(9):1041-8.

59. Skljarevski V, Zhang S, Desaiah D, Alaka KJ, Palacios S, Miazgowski T, et al. Duloxetine versus placebo in patients with chronic low back pain: a 12-week, fixed-dose, randomized, double-blind trial. J Pain. 2010:11(12):1282-90.

60. Treves R, Montane De La Roque P, Dumond JJ, Bertin P, Arnaud M, Desproges-Gotteron R. Prospective study of the analgesic action of clomipramine versus placebo in refractory low back pain and sciatica (68 cases). Rev Rhum Mal Osteoartic. 1991;58(7):549-52.

61. Urquhart DM, Wluka AE, van Tulder M, Heritier S, Forbes A, Fong C, et al. Efficacy of low-dose amitriptyline for chronic low back pain. JAMA Intern Med. 2018;178(11):1474-81.

62. Atkinson JH, Slater MA, Wahlgren DR, Williams RA, Zisook S, Pruitt SD, et al. Effects of noradrenergic and serotonergic antidepressants on chronic low back pain intensity. Pain. 1999;83(2):137-45.

63. Atkinson JH, Slater MA, Williams RA, Zisook S, Patterson TL, Grant I, et al. A placebo-controlled randomized clinical trial of nortriptyline for chronic low back pain. Pain. 1998;76(3):287-96.

64. Dickens $C$, Jayson M, Sutton C, Creed F. The relationship between pain and depression in a trial using paroxetine in sufferers of chronic low back pain. Psychosomatics. 2000;41(6):490-9.

65. Goodkin K, Gullion CM, Agras WS. A randomized, double-blind, placebocontrolled trial of trazodone hydrochloride in chronic low back pain syndrome. J Clin Psychopharmacol. 1990;10(4):269-78.

66. Gould HM, Atkinson JH, Chircop-Rollick T, D’Andrea J, Garfin S, Patel SM, et al. A randomized placebo-controlled trial of desipramine, cognitive behavioral therapy, and active placebo therapy for low back pain. Pain. 2020;161(6):1341-9.

67. Jenkins $D G$, Ebbutt $A F$, Evans $C D$. Tofranil in the treatment of low back pain. J Int Med Res. 1976;4(2):28-40.

68. Johnson K, Chatterjee N, Noor N, Crowell A, McCue R, Mackey S. Effects of duloxetine and placebo in patients with chronic low back pain. J Pain. 2011; 12(4):P49.

69. Katz J, Pennella-Vaughan J, Hetzel RD, Kanazi GE, Dworkin RH. A randomized, placebo-controlled trial of bupropion sustained release in chronic low back pain. J Pain. 2005;6(10):656-61.

70. Veroniki AA, Jackson D, Viechtbauer W, Bender R, Bowden J, Knapp G, et al. Methods to estimate the between-study variance and its uncertainty in meta-analysis. Res Synth Methods. 2016;7(1):55-79.

71. Chou R, Deyo R, Friedly J, Skelly A, Weimer M, Fu R, et al. Systemic pharmacologic therapies for low back pain: a systematic review for an American College of Physicians clinical practice guideline. Ann Intern Med. 2017;166(7):480

72. Oliveira CB, Maher CG, Pinto RZ, Traeger AC, Lin C-WC, Chenot J-F, et al. Clinical practice guidelines for the management of non-specific low back pain in primary care: an updated overview. Eur Spine J. 2018;27(11):2791-803.

73. Dworkin RH, Turk DC, Wyrwich KW, Beaton D, Cleeland CS, Farrar JT, et al. Interpreting the clinical importance of treatment outcomes in chronic pain clinical trials: IMMPACT recommendations. J Pain. 2008;9(2):105-21.

74. Lee H, Lamb SE, Bagg MK, Toomey E, Cashin AG, Moseley GL. Reproducible and replicable pain research: a critical review. Pain. 2018:159(9):1683-9.

75. Baudard M, Yavchitz A, Ravaud P, Perrodeau E, Boutron I. Impact of searching clinical trial registries in systematic reviews of pharmaceutical treatments: methodological systematic review and reanalysis of metaanalyses. BMJ. 2017:356:448.

76. Hart B, Lundh A, Bero L. Effect of reporting bias on meta-analyses of drug trials: reanalysis of meta-analyses. BMJ. 2012;344:d7202.

\section{Publisher's Note}

Springer Nature remains neutral with regard to jurisdictional claims in published maps and institutional affiliations.

Ready to submit your research? Choose BMC and benefit from:

- fast, convenient online submission

- thorough peer review by experienced researchers in your field

- rapid publication on acceptance

- support for research data, including large and complex data types

- gold Open Access which fosters wider collaboration and increased citations

- maximum visibility for your research: over $100 \mathrm{M}$ website views per year

At $\mathrm{BMC}$, research is always in progress.

Learn more biomedcentral.com/submissions 